\title{
Uma rede digital local para subsidiar o ensino e a aprendizagem de Física
}

\author{
A local digital network to support teaching and learning in Physics \\ Una red digital local para apoyar la enseñanza y el aprendizaje en Física
}

Recebido: 00/11/2021 | Revisado: 00/12/2021 | Aceito: 17/12/2021 | Publicado: 20/12/2021

\author{
Vinicius Justino Pimenta \\ ORCID: https://orcid.org/0000-0001-5728-6159 \\ Universidade Federal de Alfenas, Brasil \\ E-mail: vinicius.pimenta@sou.unifal-mg.edu.br \\ Raphael César de Souza Pimenta \\ ORCID: https://orcid.org/0000-0003-2232-3356 \\ Universidade Federal de Alfenas, Brasil \\ E-mail: raphael.pimenta@sou.unifal-mg.edu.br \\ Samuel Bueno Soltau \\ ORCID: https://orcid.org/0000-0002-7211-2533 \\ Universidade Federal de Alfenas, Brasil \\ E-mail: samuel.soltau@unifal-mg.edu.br
}

\begin{abstract}
Resumo
Diversas propostas contemporâneas de ensino de Física contemplam o uso de tecnologias computacionais. No entanto, nem todas as escolas ou ambientes não formais de ensino dispõem dos recursos e conectividade essenciais para participar e acompanhar toda a dinâmica desencadeada pela disseminação de informações via tecnologias e favorecer seu uso na aprendizagem e no ensino. Neste contexto, propomos a implementação de uma plataforma de ensino envolvendo hardware e software, criada com o objetivo de atenuar esse problema e possibilitar a conexão dos dispositivos móveis a um repositório de recursos didáticos. O método empregado caracteriza este trabalho como uma pesquisa aplicada, exploratória de abordagem qualitativa. O componente central da plataforma é o microcontrolador ESP-32 usado para gerar uma infraestrutura de rede digital mínima e de alcance restrito a um único ambiente de ensino, como o interior de salas de aula. Como prova de conceito da viabilidade do projeto, a plataforma foi testada em uma aula presencial sobre circuitos elétricos em uma turma do terceiro ano do Ensino Médio da rede pública estadual no Sul de Minas Gerais. Os resultados sobre a viabilidade na fase atual de desenvolvimento do projeto são animadores o suficiente para incentivar sua continuação, investimento em tempo e recursos para expandi-lo e melhorá-lo.
\end{abstract}

Palavras-chave: Ensino de física; Recursos computacionais; Rede local Wi-Fi.

\begin{abstract}
Several contemporary proposals for teaching physics contemplate the use of computational technologies. However, not all schools or non-formal teaching environments have the resources and essential connectivity to participate and keep up all the dynamics triggered by the dissemination of information via technologies and favor their use in learning and teaching. In this context, we propose a teaching platform involving hardware and software, created with the purpose of mitigating this problem and enabling the connection of mobile devices to a repository of didactic resources. The method used characterizes this work as applied research, exploratory with a qualitative approach. The central component of the platform is ESP-32 microcontroller used to generate a minimal, reach-out digital network infrastructure restricted to a single teaching environment, such as inside classrooms. As proof of concept of project viability, the platform was tested in a face-to-face class about electrical circuits in a third-year high class of the southern Minas Gerais state public school. The results on the feasibility in the current phase of development of the project are encouraging enough to encourage its continuation, investment in time, and resources to expand and enhance it.
\end{abstract}

Keywords: Physics teaching; Computational resources; Local Wi-Fi network.

\section{Resumen}

Varias propuestas contemporáneas para la enseñanza de la física incluyen el uso de tecnologías computacionales. Sin embargo, no todas las escuelas o ambientes de enseñanza no formal cuentan con los recursos y la conectividad esenciales para participar y acompañar todas las dinámicas desencadenadas por la difusión de la información a través de las tecnologías y favorecer su uso en el aprendizaje y la enseñanza. En este contexto, proponemos una plataforma docente que involucra hardware y software, creada con el propósito de mitigar este problema y permitir la conexión de dispositivos móviles a un repositorio de recursos didácticos. El método utilizado caracteriza este trabajo como investigación aplicada, exploratoria con enfoque cualitativo. El componente central de la plataforma es el microcontrolador ESP-32 utilizado para generar una infraestructura de red digital mínima y de alcance restringido a un solo ambiente de enseñanza, como dentro de las aulas. Como prueba de concepto de la viabilidad del proyecto, la 
plataforma fue probada en una clase presencial sobre circuitos eléctricos en una clase de tercer año de la escuela pública estatal del sur de Minas Gerais. Los resultados sobre la viabilidad en la fase actual de desarrollo del proyecto son lo suficientemente alentadores como para estimular su continuación, la inversión en tiempo y recursos para ampliarlo y mejorarlo.

Palabras clave: Enseñanza de la física; Recursos computacionales; Red Wi-Fi local.

\section{Introdução}

$\mathrm{O}$ uso de tecnologias em apoio às práticas de ensino e de aprendizagem é um tema bastante recorrente na literatura (Moran et al., 2000; Araujo \& Veit, 2004; Kenski, 2003, 2012; Araújo, 2020). Em particular os dispositivos móveis como smartphones e tablets que utilizam tecnologia embarcada para processamento de voz e de dados têm emergido como uma das mais profícuas ferramentas para subsidiar tanto as propostas de ensino em sala de aula (Aquino et al., 2020; Camargos, Ferreira \& Xavier, 2020) quanto às fundamentadas em aprendizagem ubíqua (Hightower \& Borriello, 2001; Santaella, 2013).

$\mathrm{O}$ ensino de Ciências, em especial o ensino de Física, tem se beneficiado das tecnologias móveis devido à disponibilidade de simulações, animações, cálculos e demonstrações experimentais em vídeo entre outros, criados especificamente com o propósito de servirem como recursos didáticos (Sánchez \& Selva, 2008; Barbosa et al., 2017; Sena \& Fernandes, 2018; Guedes, 2015; Oliveira Júnior \& Simões, 2021).

A onipresença da tecnologia no cotidiano da sociedade contemporânea impõe de modo cada vez mais efetivo sua inserção no ensino como um recurso potencialmente mediador da aprendizagem. $\mathrm{O}$ acesso à internet vem acompanhado de uma diversidade de recursos educacionais que podem ser utilizados nas aulas e em casa por estudantes e professores. No entanto, apesar de todo este cenário estimulante, nem todas as escolas brasileiras ou ambientes não formais de ensino dispõem dos recursos e da conectividade essencial para participar e acompanhar toda a dinâmica desencadeada pela disseminação de informações via tecnologias e favorecer seu uso no processo de aprendizagem e de ensino.

Escolas rurais ou situadas em comunidades periurbanas remotas, por exemplo, usualmente têm infraestrutura de telecomunicações precárias ou inexistentes (Belusso \& Pontarolo, 2017). Esta condição de isolamento tecnológico impede que tais espaços de se integrarem e participarem da assim denominada "sociedade da informação", bem como de se beneficiar de propostas de ensino mediadas pelas tecnologias (VanFossen \& Waterson, 2008).

Diante do cenário descrito acima, neste artigo propomos a utilização de uma plataforma de ensino envolvendo hardwares e softwares criada com o propósito de atenuar essa problemática e possibilitar a conexão de smartphones e tablets a um repositório de recursos didáticos em locais sem acesso à internet a partir do microcontrolador ESP-32. A motivação fundamental desta proposta é contribuir para que estudantes e professores antes alijados da possibilidade de uso das tecnologias disponíveis na internet em suas aulas possam ser integrados e conectados de modo a ter acesso a formas de ensino e de aprendizagem mais recentes e que de outra forma não estariam acessíveis.

\section{Aspectos Metodológicos}

O método empregado confere a este trabalho características de uma pesquisa qualitativa aplicada e, quanto aos objetivos, exploratória. Aplicada, pois "o investigador é movido pela necessidade de contribuir para fins práticos mais ou menos imediatos buscando soluções para problemas concretos" (Cervo et al., 2007, p.60) e, neste artigo, com a criação de uma rede Wi-Fi como um ponto de acesso para a conexão de dispositivos móveis e o suporte a um repositório de arquivos em locais sem acesso internet. Exploratória, por se tratar de um trabalho desenvolvido com o intuito de "desenvolver hipóteses, aumentar a familiaridade do pesquisador com um ambiente, fato ou fenômeno para a realização de uma pesquisa futura mais precisa ou modificar e clarificar conceitos" (Marconi \& Lakatos, 2003, p.188).

Seu desenvolvimento pode ser descrito em três etapas distintas: 
1. implementação da plataforma de ensino envolvendo hardwares (computador, smartphone/tablet, ESP-32, expansão para cartão SSD) e softwares.

2. criação de um repositório de arquivos e de uma rede digital local (Wi-Fi) e,

3. prova de conceito da proposta.

O componente central da plataforma de ensino é o microcontrolador ESP-32 usado para criar uma infraestrutura de rede digital mínima e de alcance restrito a um único ambiente de ensino, como o interior de salas de aula.

Para o repositório de arquivos, optamos por simulações computacionais do Phet Colorado ${ }^{1}$, uma vez que estas permitem que os estudantes possam exercitar suas intuições em relação aos fenômenos físicos e a sua descrição matemática para prever, analisar, retirar hipóteses e compreender conceitos abstratos que foram apresentados em aulas anteriores ou estudadas em livros (Sánchez \& Selva, 2008).

Deste modo, o estudante terá nas suas mãos um modelo que pode ser modificado da forma que quiser, fazer previsões e atribuir significados a conceitos estudados nas mais diferentes disciplinas (Macêdo et al., 2012), tudo isso manipulando e interagindo com um software que mostra animações na tela de um computador, smartphone ou tablet.

E para a verificar a viabilidade do projeto, utilizamos a plataforma proposta em uma aula de Física ministrada para uma turma de estudantes do terceiro ano do Ensino Médio de uma escola da rede pública estadual localizada no sul de Minas Gerais. Vale ressaltar que esta etapa teve unicamente o caráter de testar a rede em um ambiente "realista" sem qualquer pretensão de abordar os aspectos didáticos do conteúdo.

Assim posto, a seguir descrevemos a infraestrutura de software e hardware usada como plataforma de ensino para o acesso a um repositório de arquivos através do uso de smartphones pelos estudantes dentro dos limites de uma sala de aula, o processo de criação de um repositório e de uma rede Wi-Fi, bem como de um ponto de acesso para a conexão de dispositivos móveis por meio do microcontrolador ESP-32 e a experiência de aplicação da proposta.

\section{Plataforma de Ensino}

Em termos gerais, a estrutura da plataforma de ensino montada e proposta no presente estudo é composta por hardwares (computador, smartphone/tablet, ESP-32, expansão para cartão SSD) e suporta o compartilhamento de conteúdo de software formado por arquivos de diversos tipos, tais como imagens, textos, vídeos, páginas HTML entre outros.

A infraestrutura de rede digital suporta ainda o uso de simulações de fenômenos físicos (Ilabaca, 2003) e não exclui o uso em disciplinas como Química, Biologia, Matemática entre outras. No entanto, neste estudo nos restringimos ao ensino de Física e à utilização de simulações do Phet Colorado.

A base de hardware provê a conectividade mínima necessária através de protocolos, que configuram uma rede local Wi-Fi, na qual os estudantes que possuem um aparelho móvel capaz de se conectar à internet, podem acessar os conteúdos armazenados no repositório criado pelo professor, conforme esquematizado na Figura 1. Desta forma garante-se a infraestrutura indispensável que permite a utilização dos smartphones dos estudantes para desenvolver e aplicar propostas didáticas em sala de aula (Bento \& Cavalcante, 2013).

\footnotetext{
${ }^{1}$ Interactive Simulations da Universidade do Colorado disponíveis em https://phet.colorado.edu/.
} 
Figura 1: Plataforma de ensino.

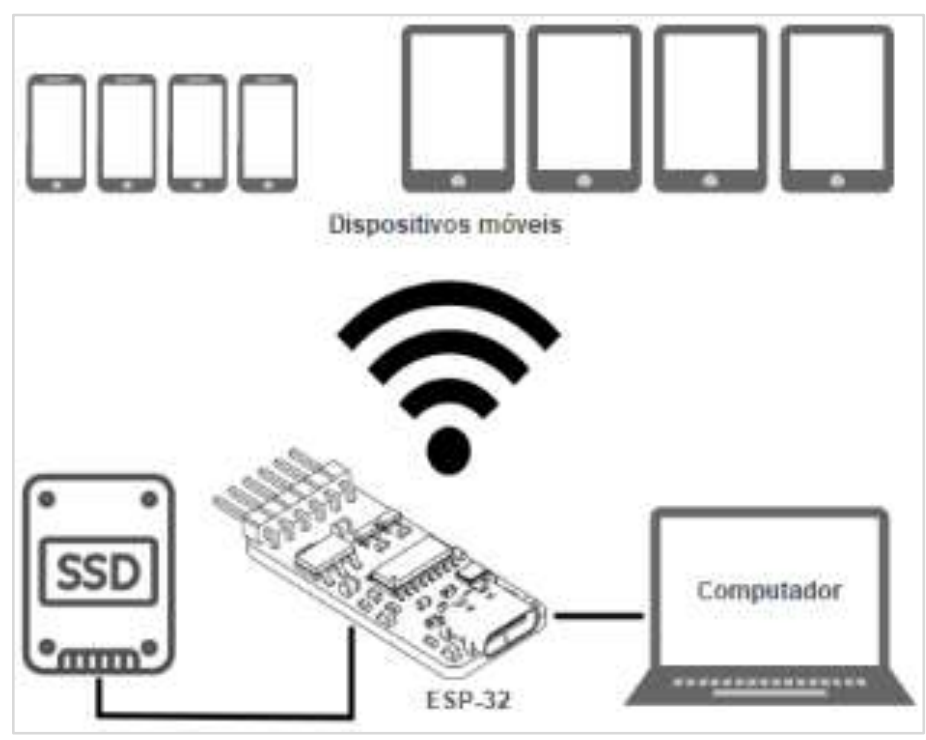

Fonte: Autores.

O núcleo de todo o projeto é o microcontrolador ESP-32, desenvolvido pela empresa Espressif ${ }^{2}$. De baixo custo e consumo de energia, constitui-se num chip que oferece Wi-Fi e Bluetooth integrados com alcance de até cinco metros, que pode ser programado através das linguagens de programação C, Python, HTML, CSS, JavaScript e permite a integração com tecnologias Arduino IDE para expandir funcionalidades e criar projetos mais flexíveis e robustos.

O microcontrolador ESP-32 possui um processador do tipo Micro Computer Unit (MCU) de 32 bits, de dois núcleos. Um dos núcleos é dedicado à conexão de alta velocidade e o outro núcleo destina-se ao desenvolvimento de aplicativos. O MCU tem uma frequência de $240 \mathrm{MHz}$ e uma memória flash integrada de 16 megabytes. Além disso, o ESP-32 suporta vários modos de controle, incluindo GPIO (General Purpose Input/Output) portas programadas, controle de toque, voz, código QR e reconhecimento facial.

Para controlar todas estas características e funcionalidades, o ESP-32 possui a plataforma de prototipagem NodeMCU32s que é utilizada para o desenvolvimento de projetos e contém um conversor de USB para alimentação VCC de 2,3V a 3,6V em corrente contínua (Espressif, 2021). Pode-se conectar o ESP-32 a uma rede Wi-Fi existente ou criar um ponto de acesso (hot spot) para conectar vários dispositivos na rede sem fio como proposto neste trabalho.

Entre os possíveis usos do ESP-32 estão a automação residencial que permite acionar equipamentos eletroeletrônicos através do smartphone ou computador implementando projetos baseados no conceito de arquitetura denominado "internet das coisas" (Internet of Things - IoT), que se refere à interconexão digital de artefatos eletrônicos cotidianos com a internet (Nath, 2017). Mas o que interessa em particular neste trabalho é a possibilidade de criar um servidor de páginas Web (Dotta \& Severo, 2020; Susany \& Rotar, 2020; Conceição et al., 2021) para, além de configurar uma rede Wi-Fi, desenvolver um repositório de arquivos.

Como o repositório de arquivos a ser implementado necessita de uma capacidade de armazenamento suficiente para comportar todos os arquivos despejados e na configuração básica o ESP-32 possui apenas 16Mb de memória Flash foi necessário expandir a memória do dispositivo com um módulo adicional de cartão SSD, como mostra a Figura 2

\footnotetext{
${ }^{2}$ https://www.espressif.com/en/products/hardware/esp32/overview.
} 
Figura 2: ESP-32, à esquerda e módulo de cartão de memória SSD, à direita.

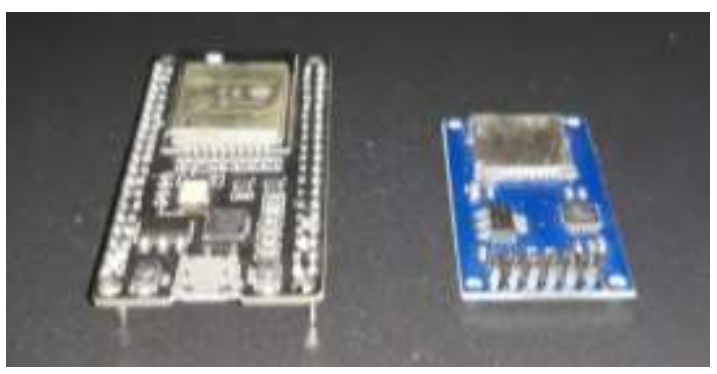

Fonte: Autores.

No repositório podem ser adicionados arquivos de mídia de vários formatos e a limitação do espaço de armazenamento passa a depender somente da capacidade do cartão de memória inserido.

Para acessar o repositório é preciso se conectar na rede Wi-Fi gerada pelo dispositivo, que é identificada pelo SSID (Service Set Identifier) e pode ser protegida por senha se necessário. Em seguida é preciso digitar no navegador de internet (internet browser) o endereço de IP (Internet Protocol) dinâmico gerado pelo ESP-32 para acessar o repositório, como em geral se faz para acessar um website da internet. O repositório funciona como se fosse um website, porém situado a rede local e, como é comum, construído em linguagem HTML. Para este trabalho foram criadas páginas simples, onde foram colocados apenas botões denominados como Home, Download, Upload e Directory conforme a Figura 3.

Figura 3: O repositório em um Desktop

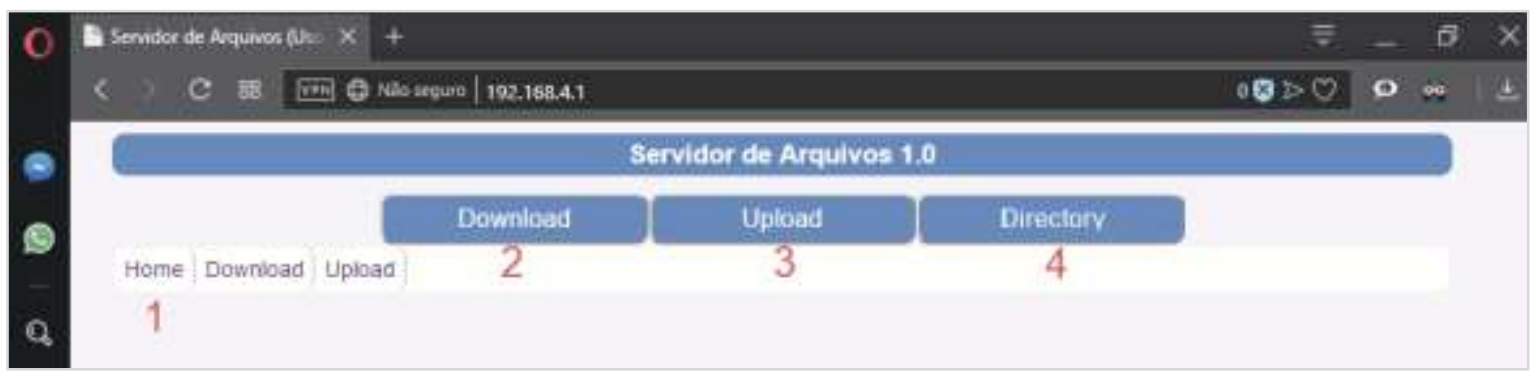

Fonte: Autores.

Onde:

1. Home, retoma a página inicial do repositório;

2. Download é usado para baixar os arquivos do repositório;

3. Upload é usado para enviar arquivos para o repositório, e;

4. Directory é a "pasta" onde se pode visualizar todos os arquivos do repositório.

Assim, caso o dispositivo cliente, i.e., o smartphone ou tablet do estudante, conectado à rede local tenha instalado o software apropriado para abrir o formato do arquivo baixado, o estudante poderá utilizar este arquivo.

Para melhor entendimento do processo de criação da rede Wi-Fi local e alimentação do repositório de arquivos, apresentamos a seguir todo o processo realizado para utilizar uma simulação do website Phet Colorado selecionada para a prova de conceito prático em sala de aula.

\section{Repositório de Arquivos e Rede Digital Local (Wi-Fi)}

Como dito anteriormente, o repositório de arquivos pode ser acessado utilizando um navegador tanto em um computador 
(Figura 3) quanto por um smartphone ou tablet como mostrado na Figura 4.

Figura 4: O repositório em um Smartphone.

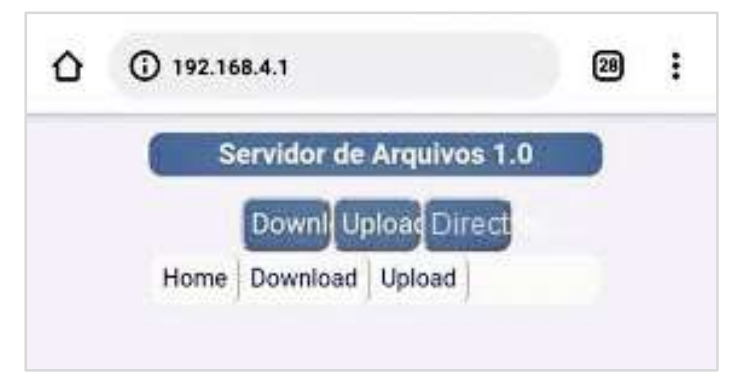

Fonte: Autores.

Na tela mostrada na Figura 5, ao clicar na aba Upload é efetuada a adição de arquivos ao repositório. São estes arquivos que contém a simulação e demais arquivos necessários para ministrar a aula e que serão acessados pelos estudantes no decorrer da aula.

Figura 5: Upload de arquivos.

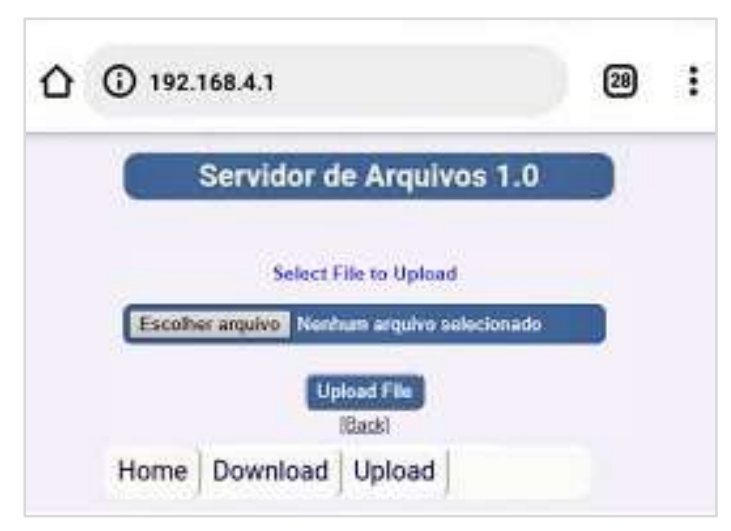

Fonte: Autores.

Após incluir todos os arquivos no repositório é possível verificar a integridade de cada um deles clicando na aba Directory conforme apresentado na Figura 6. Neste exemplo, colocamos alguns arquivos do formato HTML, como por exemplo, a simulação Kit para Montar Circuito DC - Lab Virtual, disponível no site do Phet Colorado ${ }^{3}$, utilizado posteriormente para a prova de conceito.

\footnotetext{
${ }^{3}$ Disponível em: https://phet.colorado.edu/sims/html/circuit-construction-kit-dc-virtual-lab/latest/circuit-construction-kit-dc-virtual-lab_pt_BR.html
} 
Figura 6: Diretório de arquivos.

\begin{tabular}{|l|l|l|}
\hline \multicolumn{1}{|c|}{ Servidor de Arquivos 1.0} \\
\hline \multicolumn{1}{|l|}{ SSD Card Contents } & \\
\hline Name/Type & Type File/Dir & File Size \\
\hline Dir & /System Volume & \\
\hline ISystem Volume Information/WPSettings.dat & File & 12 B \\
\hline /1.html & File & $1.35 \mathrm{MB}$ \\
\hline /circuit-construction-kit-dc-virtual-lab_pt_BR.html & File & $2.1 \mathrm{MB}$ \\
\hline Home $)$ Download Upload ) & & \\
\hline
\end{tabular}

Fonte: Autores.

Para baixar os arquivos do repositório deve-se clicar na aba Download (Figura 3 e 4) e nela digitar o nome do arquivo o qual se deseja baixar para o dispositivo cliente dos usuários, o que possibilita executá-lo posteriormente. E, para executar um arquivo desejado no dispositivo cliente, o primeiro passo é o usuário, no caso o estudante, conectar-se à rede $\mathrm{Wi}$ - $\mathrm{Fi}$, criada com a configuração do ESP-32 que neste exemplo foi renomeada como ESP32-Access-Point, Figura 7.

Figura 7: Rede para se conectar ao repositório.

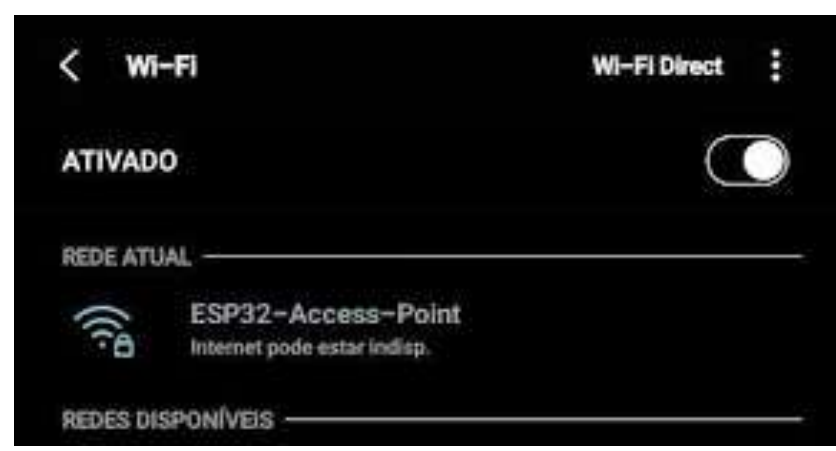

Fonte: Autores.

Para acessar a página inicial do repositório - denominada $1 . h t m l$ - é necessário digitar o IP no navegador, conforme mostrado na Figura 8.

Figura 8: Página do repositório e download.

\begin{tabular}{|c|}
\hline A 192.168.4.1/download \\
\hline Servidor de Arquivos 1.0 \\
Enter filename to download \\
1.html \\
Home \\
[Back] \\
\hline Download Upload
\end{tabular}

Fonte: Autores. 
Após digitar o nome do arquivo $(1 . \mathrm{html}$ ) e pressionar a tecla Enter (no caso do computador ou tocar com o dedo no caso dos smartphones e tablets, o arquivo é baixado no dispositivo do usuário (Figura 9).

Figura 9: Baixando o arquivo.

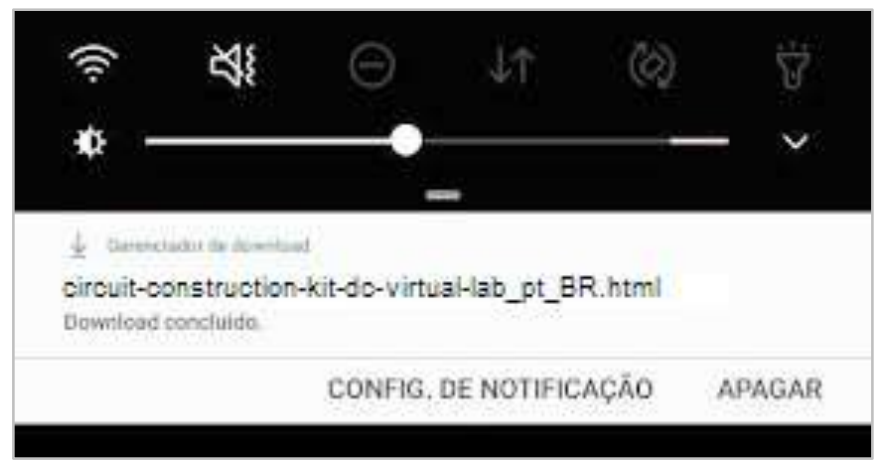

Fonte: Autores.

A Figura 10 exemplifica a execução da simulação baixada conforme aparece no smartphone.

Figura 10: Executando a Simulação no smartphone.

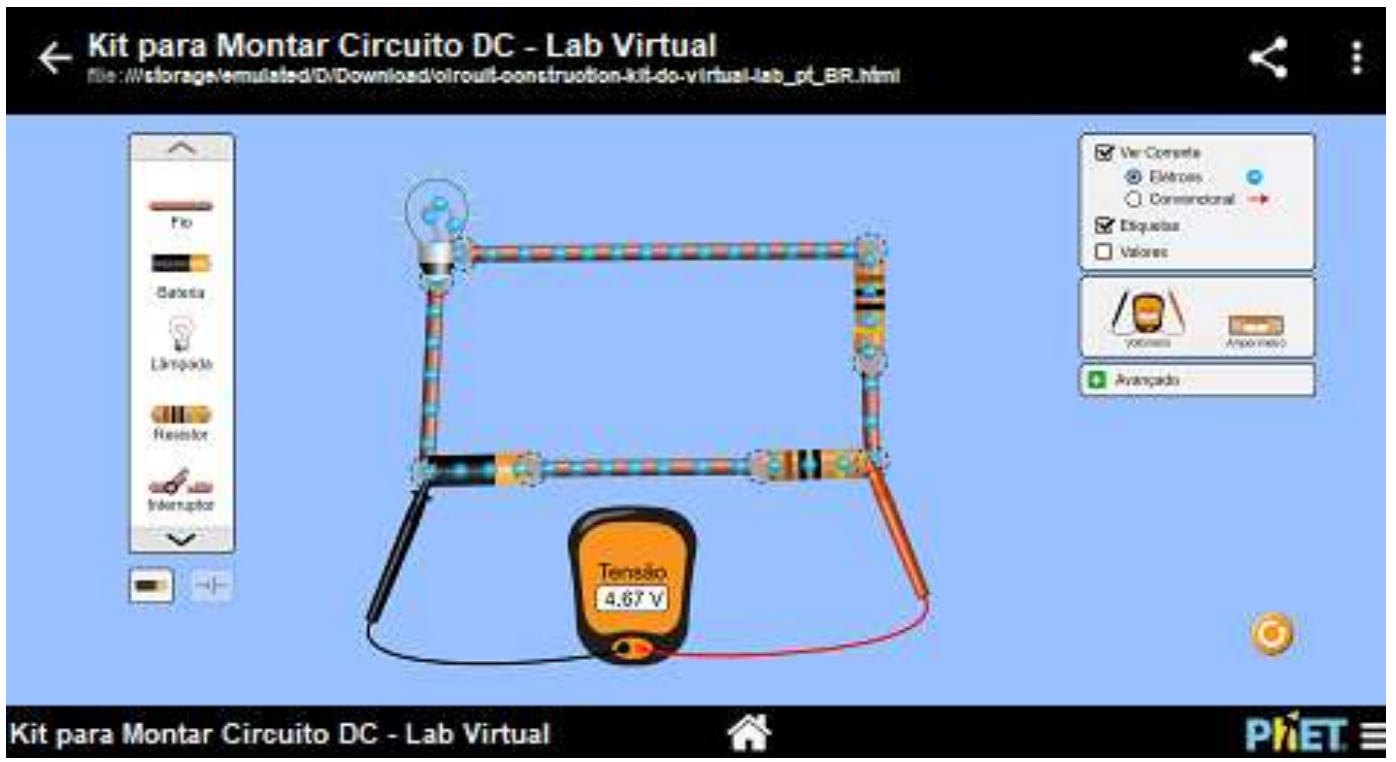

Fonte: Autores.

Com base em todos estes procedimentos, a seguir, relatamos uma experiência de aplicação da plataforma em uma aula presencial e os resultados obtidos nesta experiência.

\section{Prova de Conceito da Plataforma}

Como uma "prova de conceito" da viabilidade do projeto, relatamos a aplicação desta tecnologia em uma turma composta por trinta estudantes do terceiro ano do Ensino Médio de uma escola da rede pública estadual localizada no sul de Minas Gerais realizada durante uma aula de Física que abordou circuitos elétricos e que, graças a plataforma, pôde utilizar como recurso didático a simulação computacional de circuitos.

Para esta prova de conceito prático da plataforma em sala de aula foi acertado com o professor regente da turma a realização de uma revisão e resolução de exercícios de um conteúdo já trabalhado com os estudantes. Para tanto, selecionamos 
a simulação que aborda o tema circuitos elétricos, intitulada Kit para Montar Circuito DC - Lab Virtual, disponível no site do Phet Colorado cujo download foi realizado para compor o repositório de arquivos, como apresentado na seção anterior.

A experiência se desenvolveu em duas aulas, totalizando 100 minutos e contemplou quatro momentos distintos: $(i)$ revisão do conteúdo circuitos elétricos, (ii) apresentação da plataforma e acesso à rede Wi-Fi local gerado por ela, (iii) apresentação da simulação Kit para Montar Circuito DC - Lab Virtual e, (iv) resolução de exercícios conforme descritos a seguir.

O primeiro momento foi reservado para a revisão de conceitos básicos de eletricidade como tensão, corrente, resistência, potência elétrica, leis de Ohm e associação de resistores por serem fundamentais para a atividade que envolvia a simulação.

No momento seguinte, explicamos como funcionava a plataforma descrevendo na lousa passo a passo como acessar a rede Wi-Fi, a página inicial do repositório e realizar o Download da simulação em seus smartphones.

Considerando que o ESP-32 utilizado suporta apenas quatro conexões simultâneas estáveis, a taxa de download não foi muito alta. Em um smartphone típico um arquivo com aproximadamente $2 \mathrm{Mb}$ teve taxa de Download de $17 \mathrm{~kb} / \mathrm{s}$ e demorou cerca de dois minutos para ser baixado. Este limite de velocidade pode ser solucionado utilizando uma antena acoplada ao ESP32 que potencializa o sinal e eleva as taxas de download e upload.

Mesmo com estes problemas, nesta fase inicial de desenvolvimento da plataforma, conseguimos fazer com que os estudantes tivessem acesso a simulação em seus smartphones. A solução encontrada foi separar os estudantes em grupos conectando quatro dispositivos por vez e, apesar da diversidade de modelos, constatamos apenas problemas de compatibilidade com o arquivo HTML em dois smartphones.

Na sequência, apresentamos e explicamos o funcionamento do Kit para Montar Circuito DC - Lab Virtual e, com a simulação projetada na lousa, explicamos como usá-la em seus aparelhos smartphones (Figura 11).

Figura 11: Aula apresentada para os estudantes.

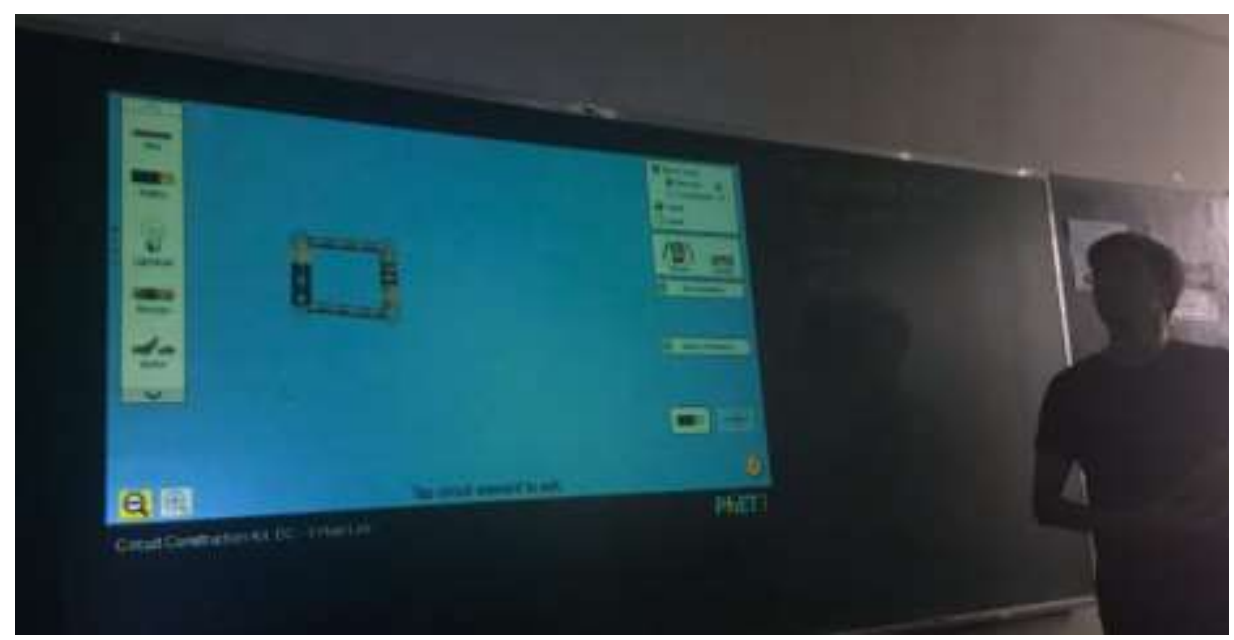

Fonte: Autores.

Após a apresentação foi solicitado aos estudantes que simulassem o mesmo circuito apresentado com o Kit para Montar Circuito DC - Lab Virtual instalado em seus smartphones e o utilizassem para calcular o valor da corrente e da diferença de potencial entre os resistores entre outras questões.

$\mathrm{Na}$ avaliação do professor regente, a plataforma mostrou-se adequada para realizar práticas envolvendo tecnologias computacionais, despertar o interesse e manter a atenção dos estudantes na aula dado o envolvimento demonstrado por eles no momento da resolução de exercícios. 


\section{Considerações Finais}

Neste artigo, descrevemos a implementação de uma plataforma de ensino que permite a criação de uma rede $W i-F i$ como um ponto de acesso para a conexão de dispositivos móveis e o suporte a um repositório de arquivos com o uso do microcontrolador ESP-32. A ideia primordial foi viabilizar práticas de ensino e de aprendizagem de conteúdos da Fisica que envolvam o uso de recursos computacionais via redes digitais em escolas e outros locais de ensino que estão situados em áreas nas quais o acesso à internet inexista ou é precário.

A implementação da plataforma permite que os professores possam disponibilizar arquivos de todos os tipos e levar ferramentas didáticas diretamente aos smartphones dos estudantes nos limites da sala de aula.

A prova de conceito mostrou que a plataforma é capaz de levar satisfatoriamente recursos computacionais para a sala de aula que simulem fenômenos físicos que antes só seriam possíveis com um computador conectado à internet ou com um arquivo baixado previamente viabilizando assim, tantas possibilidades para o ensino da Física quanto as disponíveis em escolas que possuem acesso pleno a redes digitais e a dispositivos móveis conectados.

Apesar do número de conexões simultâneas limitado apresentado pela rede $W i-F i$, no estágio de desenvolvimento atual da plataforma, foi possível realizar as atividades propostas recorrendo ao expediente de dividir a turma em grupos para efetuar o download do recurso didático utilizado. Cabe ressaltar que esta limitação pode ser facilmente contornada com o uso de uma antena adequada ou com a substituição do ESP-32 por outro microcontrolador mais robusto. Em continuidade do projeto, estudamos em versões futuras a possibilidade de ajustar alguns parâmetros do ESP-32 de forma a possibilitar mais conexões.

\section{Referências}

Aquino, C. C. F. de. et al. (2020). O Estado da Arte sobre o uso das tecnologias móveis na Educação Básica: mapeamento de trabalhos produzidos no período de 2016 a 2018. Research, Society and Development, 9(5), e63952690. https://doi.org/10.33448/rsd-v9i5.2690

Araujo, I. S., \& Veit, E. A. (2004). Uma revisão da literatura sobre estudos relativos a tecnologias computacionais no ensino de física. Revista Brasileira De Pesquisa Em Educação Em Ciências, 4(3), 5-18. https://periodicos.ufmg.br/index.php/rbpec/article/view/4069

Araujo, T. O. (2020). Tecnologias móveis na educação: reflexões e práticas. LínguaTec, 5(1), 59-80. https://doi.org/10.35819/linguatec.5(1).3352

Barbosa, C. D. et al. (2017). O uso de simuladores via smartphone no ensino de Física: O experimento de Oersted. Revista Scientia Plena, $13(1), 1-13$.

Belusso, A., \& Pontarolo, E. (2017). Uma reflexão sobre tecnologia digital nas escolas do campo como possibilidade para o desenvolvimento dos territórios camponeses. In: X Seminário Internacional sobre Desenvolvimento Regional.

Bento, M. C. M. \& Cavalcante, R. dos S. (2013). Tecnologias Móveis em Educação. O uso do smartphone na sala de aula. Educação, cultura e comunicação, $4(7), 113-120$.

Camargos, A. F. P. de, Ferreira, E., \& Xavier, G. do C. (2020). Ensino e aprendizagem de viabilidade de parques eólicos utilizando smartphone e TAS. Research, Society and Development, 9(10), e9009109309. https://doi.org/10.33448/rsd-v9i10.9309.

Cervo, A. L., Bervian, P. A., \& Silva, R. (2007). Metodologia científica. (6a ed.), Pearson Prentice Hall.

Conceição, P. S. da. et al. (2021). Monitoramento de equipamentos com comunicação bluetooth e wifi. Revista Brasileira de Mecatrônica, 4(1), 72-89.

Dotta, F. P., \& Severo, L. S. (2020). Estudos e aplicações iniciais do processador ESP-32 em sistemas de internet das coisas IOT. Anais do Salão Internacional de Ensino, Pesquisa e Extensão, 12(2). Recuperado de https://periodicos.unipampa.edu.br/index.php/SIEPE/article/view/107509

Espressif (2021). Esp-32. Recuperado de https://www.espressif.com/en/products/socs/esp32.

Guedes, A. G. (2015). Estudo de ondas estacionárias em uma corda com a utilização de um aplicativo gratuito para smartphones. Revista Brasileira de Ensino de Física, 37(2). 2502-1. http://dx.doi.org/10.1590/S1806-11173721666

Hightower, J., \& Borriello, G. (2001). Location systems for ubiquitous computing. Computer, 34(8), 57-66.

Ilabaca, J. S. (2003). Integración curricular de tics concepto y modelos. Revista enfoques educacionales, 5(1), 51-65.

Kenski, V. M (2003). Aprendizagem mediada pela tecnologia. Revista diálogo educacional, 4(10), 1-10.

Kenski, V. M. (2012). Educação e tecnologias: o novo ritmo da informação. (8a ed.), Papirus. 
Research, Society and Development, v. 10, n. 17, e170101723980, 2021

(CC BY 4.0) | ISSN 2525-3409 | DOI: http://dx.doi.org/10.33448/rsd-v10i17.23980

Macêdo, J. A. de, Dickman, A. G., \& Andrade, I. S. F. de. (2012). Simulações computacionais como ferramentas para o ensino de conceitos básicos de eletricidade. Caderno Brasileiro de Ensino de Física, 562-613.

Marconi, M. A., \& Lakatos, E. M. (2003). Fundamentos de Metodologia Cientifica (5a ed.) Atlas.

Moran, J. M., Masseto, M. T. \& Behrens, I. A. (2000). Novas tecnologias e mediação pedagógica. (10a ed.) Papirus.

Nath, S. V. (2017). IoT Architecture. In: Geng, H. (ed.). Internet of Things and Data Analytics Handbook. Wiley.

Oliveira Júnior, A. J. de., \& Simões, R. P. (2021). Desenvolvimento de um aplicativo Android utilizando a classe FlingAnimation para abordagem de conceitos de cinemática. Research, Society and Development, 10(1), e28410111710. https://doi.org/10.33448/rsd-v10i1.11710.

Sánchez, M. A., \& Selva, V. S. (2008). Animaciones modellus para las clases de fisica. Revista Española de Física, 22(3). 52-57

Santaella, L. (2013). Comunicação ubíqua: repercussões na cultura e na educação. Paulus (Coleção comunicação).

Sena, C. G., \& Fernandes, G. W. R. (2018). Tecnologias móveis: uma proposta didática de Física para o uso do aplicativo "Física Lab Resistores". Experiências em Ensino de Ciências, 13(5), 352-376.

Susany, R., \& Rotar, R. (2020). Remote Control Android-based Applications for a Home Automation implemented with Arduino Mega 2560 and ESP 32. Technium: Romanian Journal of Applied Sciences and Technology, 2(2), 1-8. https://doi.org/10.47577/technium.v2i2.231

VanFossen, P. J., \& Waterson, R. A. (2008). "It is just easier to do what you did before...”: An Update on Internet Use in Secondary Social Studies Classrooms in Indiana. Theory \& Research in Social Education, 36(2), 124-152. https://doi.org/10.1080/00933104.2008.10473369 